\title{
La epigenética en la diabetes mellitus tipo 2: una enfermedad crónica no transmisible
}

\section{Epigenetics in type 2 diabetes mellitus: a non-communicable chronic disease}

\author{
Alexandro J. Martagón-Rosado $1,2 *$
}

${ }^{1}$ Unidad de Investigación de Enfermedades Metabólicas, Instituto Nacional de Ciencias Médicas y Nutrición Salvador Zubirán; ${ }^{2}$ Escuela de Medicina y Ciencias de la Salud, Tecnologico de Monterrey. Ciudad de México, México

\section{RESUMEN}

La diabetes mellitus tipo 2 continúa siendo una pandemia sin aparente solución. Estudios realizados en las últimas dos décadas han encontrado una relación importante entre la epigenética y la diabetes mellitus tipo 2, determinando que el ambiente en el que el individuo se desarrolla (incluso en la etapa prenatal) tiene una influencia importante en el futuro desarrollo de diversas patologías. La metilación del ADN, la modificación de histonas y los ARN no codificantes son los principales reguladores de la epigenética. A diferencia del genoma, el epigenoma es potencialmente reversible y se puede corregir por medio de diferentes intervenciones que podrían modificar el curso de esta enfermedad. A pesar de lo anterior, la epigenética presenta importantes limitantes que retrasan su estudio; aunado a los estudios genéticos, la proteómica y la metabolómica, la epigenética es una alternativa más para la identificación de biomarcadores que permitan una predicción para el desarrollo de la diabetes mellitus tipo 2 .

Palabras clave: Diabetes mellitus tipo 2. Epigenómica. Enfermedad crónica no transmisible.

\section{ABSTRACT}

Type 2 diabetes mellitus continues to be a pandemic without apparent solution. Studies performed in the last couple of decades have found an important relationship between epigenetics and type 2 diabetes mellitus, determining that the environment in which an individual develops, even during the prenatal stage has an important influence in the future development of diverse pathologies. DNA methylation, histone modification and non-coding RNA's are the main regulators of epigenetics. In contrast to the genome, the epigenome is potentially reversible and may be corrected through different interventions that could modify the course of this disease. Despite of this, epigenetics present certain limitations that delay its study; along with genetics, proteomics and metabolomics, epigenetic is one more alternative for the identification of biomarkers that may allow a prediction for the development of type 2 diabetes mellitus.

Key words: Diabetes mellitus type 2. Epigenomics. Noncommunicable diseases.
Correspondencia:

*Alexandro J. Martagón-Rosado

E-mail: alexandro.martagonraincmnsz.mx
Fecha de recepción: 24-05-2021

Fecha de aceptación: 10-06-2021

DOI: 10.24875/ALAD. 21000013
Disponible en internet: 29-09-2021 Rev ALAD. 2021;11:74-81

2248-6518 / ๑ 2021 Asociación Latinoamericana de Diabetes. Publicado por Permanyer. Este es un artículo open access bajo la licencia CC BY-NC-ND (http://creativecommons.org/licenses/by-nc-nd/4.0/). 


\section{INTRODUCCIÓN}

Una de las pandemias del siglo XXI, la diabetes mellitus tipo 2 (DM2), se caracteriza por una función alterada de las células beta y del metabolismo de la glucosa en el hígado; un estado en el que los tejidos presentan una respuesta disminuida a la insulina y una secreción alterada de esta ${ }^{1,2}$. La evidencia ha demostrado que la DM2 no solo está causada por factores genéticos y del estilo de vida, sino también por factores que ocurren en el desarrollo temprano del individuo y que lo llevan a padecer enfermedades crónicas no transmisibles ${ }^{3}$. A partir de la década de los años 2000, la epigenética tomó gran auge como parte de la ciencia más novedosa y avanzada para el estudio de las enfermedades, desde un nivel mucho más profundo, incluida la DM2.

La epigenética se define como una alteración determinada por fenómenos ambientales en la expresión de genes, sin que estos cambios sean evidentes en la secuencia de $A D N{ }^{4,5}$. En la actualidad, la incidencia de DM2 continúa en aumento en Latinoamérica y en el mundo, y diversas investigaciones han determinado que existen mecanismos epigenéticos implicados en el control de la expresión de genes que funcionan como un vínculo entre algunas condiciones adversas en la vida temprana del individuo y el desarrollo de enfermedades crónicas como la DM2 en etapas más avanzadas de la vida 6 . En este texto se expondrá la relación que tiene la epigenética con la DM2 y sus causas, así como implicaciones en prevención, diagnóstico y tratamiento.

\section{¿CÓMO SE RELACIONAN LA EPIGENÉTICA Y LA DIABETES MELLITUS TIPO 2?}

\section{El papel de la epigenética en el desarrollo de la diabetes mellitus tipo 2}

La epigenética ha sido postulada como una explicación para el origen del desarrollo de la salud y la enfermedad (DOHaD: developmental origin of health and disease $)^{7}$ y que se relaciona con el entorno que existe desde la concepción, el entorno prenatal y posnatal, así como ciertas exposiciones maternas o paternas independientes, incluso antes de la concepción, que se pueden transmitir epigenéticamente de manera transgeneracional ${ }^{8}$.

Los factores ambientales como la dieta, la actividad física, el ritmo circadiano, el estrés y la temperatura, entre otros factores, pueden tener efectos sobre la expresión de genes por medio de mecanismos epigenéticos, que determinarán las respuestas celulares y su adaptación a su entorno9. Por este motivo, un estado metabólico alterado puede afectar al epigenoma y al fenotipo de diferentes órganos, y así contribuir al desarrollo de la DM2 y sus múltiples complicaciones ${ }^{10}$ (Fig. 1).

\section{La regulación epigenética}

La regulación epigenética se da por metilación del $A D N$, por modificaciones en las histonas y por los ARN no codificantes ${ }^{11}$, en donde una disrupción en este equilibrio puede contribuir al desarrollo de diferentes patologías, desde la obesidad hasta la $\mathrm{DM}_{2}{ }^{12}$. Para determinar si la epigenética realmente contribuye a la $\mathrm{DM} 2$, se deben identificar las alteraciones epigenéticas en pacientes que viven con DM2 contra pacientes sin DM2; una vez que se han identificado dichas alteraciones se debe buscar si son causa o consecuencia y su rol patogénico en diferentes órganos asociados a la enfermedad ${ }^{12}$.

Los primeros estudios en donde se analizó la metilación en ADN fueron llevados a cabo en genes candidatos para DM2 (INS, PDX1, PPARGC1A y GLP1R) en islotes pancreáticos humanos de donadores con DM2 y controles sanos. En dichos estudios se identificó un aumento en la metilación de ADN y una 


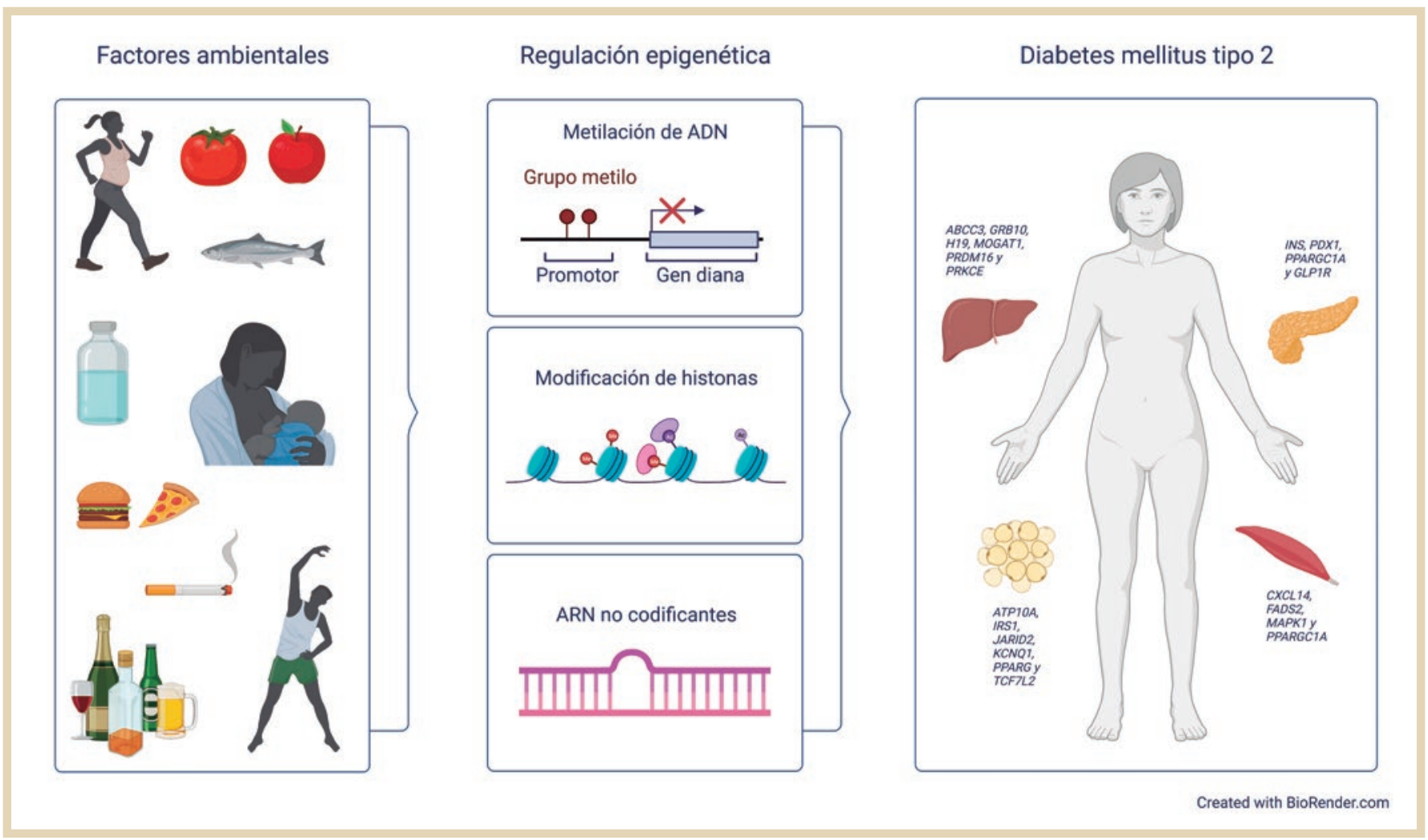

Figura 1. La influencia de los fenómenos ambientales genera cambios epigenéticos que intervienen en el desarrollo de la diabetes mellitus tipo 2.

disminución en la expresión de estos genes asociados con una secreción de insulina alterada ${ }^{13-15}$, además se demostró que la glucosa elevada y la hemoglobina glucosilada (HbA1c) también aumentan directamente la metilación de ADN en estos genes ${ }^{15}$.

Mediante arreglos Illumina ${ }^{\circledR}$ no solo se ha estudiando la metilación de ADN en islotes pancreáticos, sino también en tejido adiposo (ATP10A, IRS1, JARID2, KCNQ1, PPARG y TCF7L2), hígado ( $A B C C 3$, GRB10, H19, MOGAT1, PRDM16 y PRKCE) y músculo esquelético ( $C X C L 14, F A D S 2$, MAPK1 y PPARGC1A) en sujetos que viven con y $\sin \mathrm{DM}_{2}$, identificando múltiples sitios CpG con metilación de ADN alterada en tejidos blanco de pacientes con DM2, confirmando su patogenicidad en esta enfermedad ${ }^{16-20}$. Aun cuando estos resultados fueron importantes, su efecto es modesto, lo cual Ling y Rönn definen como esperado, puesto que la $\mathrm{DM} 2$ es una enfermedad compleja, poligénica y multifactorial que no se puede explicar por uno o incluso varios factores asocia$\operatorname{dos}^{12}$. Es claro que incluso el conocimiento de las variantes genéticas asociadas al desarrollo de DM2 solo explican del 5-10\% de la heredabilidad de esta ${ }^{21}$. Asimismo, se han llevado a cabo estudios de asociación del epigenoma completo, en donde se han identificado posiciones variables de metilación (MVP) asociadas con DM2 incidente. En un estudio realizado por Cardona, et al. se identificaron 15 nuevos MVP, además de tres que ya habían sido confirmados, lo que lleva a un total de 18 MVP asociados de manera robusta a DM2 incidente y prevalente en estudios independientes ${ }^{22}$. Estos MVP se han relacionado con vías metabólicas asociadas con el metabolismos de la glucosa y la obesidad; sin embargo, lo más interesante es que ninguno de los genes implicados en estos MVP habían sido identificados en estudios de asociación del genoma, lo cual indica 
que los estudios de metilación del ADN son una estrategia novedosa y útil para la identificación de nuevos mecanismos involucrados en el desarrollo de la $\mathrm{DM} 2^{22}$.

Por otro lado, también se han estudiado las modificaciones permanentes en las histonas, denominadas «memoria metabólica», en donde se propone que los niveles de glucosa continuamente elevados generan modificaciones permanentes en las histonas, incluso cuando la glucosa se ha normalizado ${ }^{8}$. Lo anterior ha sido demostrado en estudios como el United Kingdom Prospective Diabetes Studies (UKPDS) y el Diabetes Control and Complications Trial (DCCT), en donde se ha demostrado que un control de glucosa inadecuado desde el diagnóstico se vincula con mayor frecuencia de complicaciones décadas después, aun cuando se haya logrado un control glucémico en periodos intermedios ${ }^{23,24}$. Aquí la importancia de un control temprano y oportuno de la glucemia en pacientes diagnosticados con DM2 para mejorar la expectativa de vida en pacientes con esta enfermedad ${ }^{25}$. Es importante recordar que la hiperglucemia en madres diabéticas también es una causa de modificaciones epigenéticas durante el periodo embrionario para el producto, con potencial efecto a largo plazo ${ }^{26}$.

Por último, los ARN no codificantes son moléculas de ARN que no codifican proteínas, pero que regulan la expresión de genes a nivel transcripcional y postranscripcional ${ }^{27}$. Los ARN no codificantes mayormente implicados en los cambios epigenéticos son los micro-ARN (miARN), los ARN de interferencia pequeños (ARNip), los ARN con interacción piwi (piRNA) y los ARN largos no codificantes (IncRNA) ${ }^{28}$. En estudios de miARN en plasma de pacientes con diabetes se han evidenciado niveles bajos de diversos miARN involucrados en procesos inflamatorios, incluidos los miR-15a, miR-21, miR-20b y miR-29b. Específicamente el miR-15a se mostró disminuido en células endoteliales retinales murinas bajo condiciones de glucosa elevada, con una función antiinflamatoria ${ }^{29}$. En cuanto a los miARN $20 b$ y 29b, estudios han demostrado que tienen como blanco a proteínas involucradas en la diferenciación de células productoras de citocinas, las células T colaboradoras, Th17 y Th1, respectivamente ${ }^{30,31}$. Lo interesante de estos estudios fue que los niveles bajos de miR-15a y de miR-29b siempre precedieron a la manifestación de $\mathrm{DM}^{32}$.

En la actualidad se ha aceptado que mecanismos como la inflamación crónica, el estrés oxidativo, los productos de glucosilación avanzada y los cambios epigenéticos están involucrados en la «memoria metabólica» 25 .

\section{La epigenética en la prevención, el diagnóstico y el tratamiento}

Hoy en día se sabe que la posibilidad de que existan ciertos epigenotipos puede ayudar al abordaje preventivo y manejo del riesgo de padecer $\mathrm{DM} 2$ y sus complicaciones 33 . En un estudio llevado a cabo por Godfrey, et al. se demuestra que el perfil epigenético al nacimiento puede predecir el nivel de adiposidad en la infancia y la exposición nutricional a la que estuvo expuesto durante su vida prenatal. En este estudio analizaron al receptor retinoide, quien controla el metabolismo del tejido adiposo y la sensibilidad a la insulina, y pudieron observar en dos cohortes independientes cómo la metilación del promotor de este receptor se correlaciona con el nivel de adiposidad a los 6 y 9 años de edad. Además, probaron que la ingesta de carbohidratos de la madre durante el primer trimestre del embarazo también se encontraba relacionado con la metilación del mismo promotor, la adiposidad del producto y su riesgo de desarrollar DM2 más adelante en la vida34. Asimismo, se ha estudiado a nivel 
epigenético un gen íntimamente relacionado con la obesidad y la DM2, el gen FTO. Por medio de estos estudios se demostró en su momento que el bajo nivel de metilación de FTO era un marcador temprano de DM2; teniendo este nivel de metilación un poder predictivo incluso mayor que el de las variantes genéticas relacionadas con él35.

Avances recientes enfocados en la nutrición de precisión han descubierto que la dieta de los individuos puede incrementar el riesgo de desarrollar DM2 al interactuar con variantes genéticas específicas, modificando a su vez las características epigenéticas y la expresión de genes con la subsecuente alteración de las vías metabólicas ${ }^{36}$. Por ejemplo, se ha observado en ratas que una dieta baja en proteína durante el embarazo puede disminuir la acetilación de la histona $\mathrm{H} 3$ del promotor de NR1H3, lo cual silencia su expresión e incrementa el riesgo de $\mathrm{DM} 2$ en el producto ${ }^{37}$. Por otro lado, en estudios de metilación de ADN hepático materno murino se ha visto que la restricción dietaria de cromo resulta en genes hipermetilados involucrados en la señalización de la insulina, los cuales se encuentran regulados hacia la baja y, por consiguiente, promueven la $\mathrm{DM} 2^{38}$, mientras que la deficiencia de magnesio y de calcio igualmente incrementan el riesgo de desarrollar $\mathrm{DM}_{2}$ al inducir metilaciones aberrantes de ADN en genes relacionados con el metabolismo de los glucocorticoi$\operatorname{des}^{39,40}$.

Asimismo, se ha comprobado el efecto que tiene la actividad física sobre la metilación del ADN en músculo esquelético de familiares de primer grado de pacientes con $\mathrm{DM} 2$, en donde mediante una intervención de seis meses de ejercicio pudieron detectar asociaciones con cambios epigenéticos ${ }^{41}$. En el estudio anterior se observó una disminución en la metilación de ADN de RUNX1 y MEF2A, que son factores de transcripción involucrados en el entrenamiento físico, en THADA, que está asociado con DM2, y en NDUFC2, que es parte de la cadena respiratoria y que mostró disminución en la metilación de ADN posterior a la actividad física ${ }^{41}$.

Por otro lado, y de manera muy importante, se ha observado que medicamentos de uso común en pacientes con $\mathrm{DM}_{2}$, como las estatinas, pueden tener efectos en el epigenoma por medio de la acetilación de histonas y la modificación en la regulación hacia ambos sentidos de los miARN principalmente ${ }^{42}$. Ochoa-Rosales, et al. han determinado una asociación entre el uso de estatinas y la metilación de ADN; a mayor dosis de estatinas, menor expresión de $A B C G 1$, resultando en un incremento en niveles de glucosa, insulina HbA1c, lipoproteínas de alta densidad y triglicéridos ${ }^{43}$. Lo anterior representa un rol fundamental en los efectos adversos y pleiotrópicos que generan estos medicamentos, no solo en la $\mathrm{DM} 2$, sino también se involucran en otros procesos como los de ateroesclerosis y cáncer ${ }^{42}$.

Uno de los puntos más importantes que destacar es que, así como la dieta de los individuos puede incrementar el riesgo para desarrollar DM2 al interactuar la genética y la epigenética, de igual forma la epigenética lo puede disminuir al ser reversible. Las intervenciones dietarias son capaces de modificar la expresión y las modificaciones epigenéticas de los genes involucrados en las rutas metabólicas, mientras que los perfiles de expresión y los marcadores epigenéticos pueden funcionar como predictores de respuestas personalizadas ${ }^{36}$.

La regulación de la expresión de genes es un determinante crucial en el desarrollo de las enfermedades y las modificaciones epigenéticas juegan un rol esencial en esta regulación. El cuerpo humano consta de más de 200 tipos de células diferentes que consisten esencialmente de la misma secuencia 
genómica; sin embargo, el epigenoma difiere importantemente entre individuos y células, por lo que este último muestra un dinamismo significativamente diferente ${ }^{44}$.

\section{DISCUSIÓN}

Los cambios en la expresión de genes pueden afectar desde las células y los tejidos, hasta organismos completos en donde estas alteraciones pueden persistir durante toda la vida y pasar de manera transgeneracional por herencia epigenética ${ }^{8}$.

La información adquirida en el último par de décadas ha permitido determinar que la exposición al ambiente, incluso desde la etapa prenatal, puede conllevar modificaciones epigenéticas que confieran riesgo futuro para desarrollar DM2. Diferentes estudios de GWA (genome wide association) y EWA (epigenome wide association) han identificado genes y blancos nuevos en las rutas metabólicas que requieren mayor investigación para determinar su relación con la resistencia a la insulina en tejidos periféricos, así como la falla de los islotes pancreáticos ${ }^{45}$. Esto último ha sido estudiado con mayor detalle en los último años, dando lugar al concepto de desdiferenciación de las células beta pancreáticas, es decir, estas células no solo mueren por apoptosis secundaria al daño celular ya conocido, sino que pierden su capacidad de expresión de genes, así como sufren un aumento en la regulación de genes que generalmente no se expresarían en células beta maduras ${ }^{46}$. La desdiferenciación de las células beta ha sido identificada como un cambio epigenético generado por la alteración en el perfil de transcripción de las células beta, principalmente en el de los genes del complejo Polycomb PRC2 ${ }^{47}$. En dicho estudio, Lu, et al. evidencian la importancia de la regulación transcripcional de PCR2 para el mantenimiento a largo plazo de la identidad de las células beta y como su desequilibrio desencadena un proceso de desdiferenciación y $\mathrm{DM}^{47}$.

Sin embargo, existen limitaciones importantes para el estudio de la epigenética. El acceso longitudinal a tejidos específicos en humanos es una de ellas; sin embargo, la manera más factible de realizar estos estudios es en sangre de cordón umbilical, placenta o sangre total; resultados que después se extrapolan a efectos epigenéticos derivados de un ambiente intrauterino adverso y la programación de los tejidos blanco ${ }^{11}$. Bansal, et al. han sugerido que los cambios epigenéticos son tan específicos para células y tejidos, que extrapolar sus resultados hacia objetivos más grandes puede resultar erróneo y no permitir realmente determinar su causalidad ${ }^{11}$. Aun así, grandes avances se han realizado y evidencia contundente se ha generado que permite comprender la epigenética y contextualizarla en enfermedades como la DM2 y el cáncer, entre otras.

Hoy en día se propone la identificación de anormalidades epigenéticas y su corrección mediante herramientas avanzadas de edición genética, epigenética y transcripcional, mediante las cuales se podrían resolver cuestiones involucradas en mecanismos moleculares dentro de las rutas metabólicas, su progresión en el tiempo y su reversibilidad ${ }^{48}$. Lo anterior, por supuesto, ha originado desde un inicio perspectivas éticas, sobre la legalidad, el acceso a dichas herramientas y el alcance de dichas implicaciones 49 .

\section{CONCLUSIÓN}

La DM2 es una enfermedad crónica que sin duda colapsa en diferentes grados los sistemas de salud de todos los países en los diferentes niveles de desarrollo. Es prioritario hacer modificaciones en las políticas públicas que permitan limitar el ambiente 
obesogénico en el que los seres humanos se desarroIlan. Los cambios epigenéticos inducidos por el ambiente, incluso antes de nacer, tienen repercusiones graves en la salud de la población y deben prevenirse inicialmente mediante la alimentación. Es importante cuestionarnos si la DM2 es realmente transmisible o no, pues posiblemente y desde el punto de vista epigenético, no solo se trasmite de abuelos a padres e hijos, sino que se transmite por una herencia transgeneracional que va más allá de nuestro conocimiento. Este hecho debe ser un incentivo para que quienes viven hoy, mejoren sus hábitos alimenticios, de actividad física y su estilo de vida, de manera que hereden a su descendencia inmediata y en el largo plazo una estructura epigenética saludable o que se logre ir corrigiendo aquello que ya fue heredado transgeneracionalmente y que cada individuo potencialmente desconoce.

Queda un camino largo por recorrer en cuanto a la búsqueda de biomarcadores para DM2 más allá de la genética, sin embargo, se continúa avanzando rápidamente en el aspecto de la epigenética, así como importantemente en el área de la proteómica y metabolómica para que eventualmente permitan realizar una predicción robusta con respecto al desarrollo de la DM2.

\section{FINANCIAMIENTO}

Este estudio ha sido financiado por la Unidad de Investigación de Enfermedades Metabólicas (Instituto Nacional de Ciencias Médicas y Nutrición Salvador Zubirán).

\section{CONFLICTO DE INTERESES}

El autor declara no tener conflictos de intereses relacionados con el tema expuesto en este manuscrito.

\section{RESPONSABILIDADES ÉTICAS}

Protección de personas y animales: Los autores declaran que para esta investigación no se han realizado experimentos en seres humanos ni en animales.

Confidencialidad de los datos: Los autores declaran que en este artículo no aparecen datos de pacientes.

\section{Derecho a la privacidad y consentimiento informa-} do: Los autores declaran que en este artículo no aparecen datos de pacientes.

\section{BIBLIOGRAFÍA}

1. Wilcox G. Insulin and insulin resistance. Clin Biochem Rev. 2005; 26(2):19-39.

2. Skyler JS, Bakris GL, Bonifacio E, Darsow T, Eckel RH, Groop L, et al. Differentiation of diabetes by pathophysiology, natural history, and prognosis. Diabetes. 2017;66(2):241-55.

3. Vaiserman A, Lushchak O. Developmental origins of type 2 diabetes: Focus on epigenetics. Ageing Res Rev. 2019;55:100957.

4. Bird A. Perceptions of epigenetics. Nature. 2007;447(7143):396-8.

5. Singh R, Chandel S, Dey D, Ghosh A, Roy S, Ravichandiran V, et al. Epigenetic modification and therapeutic targets of diabetes mellitus. Biosci Rep. 2020;40(9):BSR20202160.

6. Bianco-Miotto T, Craig JM, Gasser YP, van Dijk SJ, Ozanne SE. Epigenetics and DOHaD: from basics to birth and beyond. J Dev Orig Health Dis. 2017;8(5):513-9.

7. Gluckman PD, Hanson MA, Buklijas T, Low FM, Beedle AS. Epigenetic mechanisms that underpin metabolic and cardiovascular diseases. Nat Rev Endocrinol. 2009;5(7):401-8.

8. Szabó M, Máté B, Csép K, Benedek T. Epigenetic modifications linked to T2D, the heritability gap, and potential therapeutic targets. Biochem Genet. 2018;56(6):553-74.

9. Ling C, Groop L. Epigenetics: A molecular link between environmental factors and type 2 diabetes. Diabetes. 2009;58(12):2718-25.

10. Reddy MA, Zhang E, Natarajan R. Epigenetic mechanisms in diabetic complications and metabolic memory. Diabetologia. 2015;58(3):443-55.

11. Bansal A, Simmons RA. Epigenetics and developmental origins of diabetes: correlation or causation? Am J Physiol-Endocrinol Metab. 2018;315(1):E15-28.

12. Ling C, Rönn T. Epigenetics in human obesity and type 2 diabetes. Cell Metab. 2019;29(5):1028-44

13. Hall E, Dayeh T, Kirkpatrick CL, Wollheim CB, Dekker Nitert M, Ling C. DNA methylation of the glucagon-like peptide 1 receptor (GLP1R) in human pancreatic islets. BMC Med Genet. 2013;14(1):76.

14. Ling C, del Guerra S, Lupi R, Rönn T, Granhall C, Luthman H, et al. Epigenetic regulation of PPARGC1A in human type 2 diabetic islets and effect on insulin secretion. Diabetologia. 2008;51(4):615-22.

15. Yang BT, Dayeh TA, Kirkpatrick CL, Taneera J, Kumar R, Groop L, et al. Insulin promoter DNA methylation correlates negatively with insulin gene expression and positively with HbA1c levels in human pancreatic islets. Diabetologia. 2011;54(2):360-7. 
16. Abderrahmani A, Yengo L, Caiazzo R, Canouil M, Cauchi S, Raverdy V, et al. Increased hepatic PDGF-AA signaling mediates liver insulin resistance in obesity-associated type 2 diabetes. Diabetes. 2018;67(7): 1310-21.

17. Kirchner H, Sinha I, Gao H, Ruby MA, Schönke M, Lindvall JM, et al. Altered DNA methylation of glycolytic and lipogenic genes in liver from obese and type 2 diabetic patients. Mol Metab. 2016;5(3):171-83.

18. Nilsson E, Jansson PA, Perfilyev A, Volkov P, Pedersen M, Svensson MK, et al. Altered DNA methylation and differential expression of genes influencing metabolism and inflammation in adipose tissue from subjects with type 2 diabetes. Diabetes. 2014;63(9):2962-76.

19. Nilsson E, Matte A, Perfilyev A, de Mello VD, Käkelä P, Pihlajamäki J, et al. Epigenetic alterations in human liver from subjects with type 2 diabetes in parallel with reduced folate levels. J Clin Endocrinol Metab. 2015;100(11):E1491-501.

20. Ribel-Madsen R, Fraga MF, Jacobsen S, Bork-Jensen J, Lara E, Calvanese V, et al. Genome-wide analysis of DNA methylation differences in muscle and fat from monozygotic twins discordant for type 2 diabetes. PLoS One. 2012;7(12):e51302.

21. Schwenk RW, Vogel H, Schürmann A. Genetic and epigenetic control of metabolic health. Mol Metab. 2013;2(4):337-47.

22. Cardona A, Day FR, Perry JRB, Loh M, Chu AY, Lehne B, et al. Epigenome-wide association study of incident type 2 diabetes in a British population: EPIC-Norfolk Study. Diabetes. 2019;68(12):2315-26.

23. Villeneuve LM, Natarajan R. The role of epigenetics in the pathology of diabetic complications. Am J Physiol-Ren Physiol. 2010;299(1): F14-25.

24. Miao F, Chen Z, Genuth S, Paterson A, Zhang L, Wu X, et al. Evaluating the role of epigenetic histone modifications in the metabolic memory of type 1 diabetes. Diabetes. 2014;63(5):1748-62.

25. Pang M, Li Y, Gu W, Sun Z, Wang Z, Li L. Recent advances in epigenetics of macrovascular complications in diabetes mellitus. Heart Lung Circ. 2021;30(2):186-96.

26. Salbaum JM, Kappen C. Diabetic embryopathy: A role for the epigenome? Birt Defects Res A Clin Mol Teratol. 2011;91(8):770-80.

27. Holoch D, Moazed D. RNA-mediated epigenetic regulation of gene expression. Nat Rev Genet. 2015;16(2):71-84.

28. Kaikkonen MU, Lam MTY, Glass CK. Non-coding RNAs as regulators of gene expression and epigenetics. Cardiovasc Res. 2011;90(3):430-40.

29. Ye E-A, Liu L, Jiang Y, Jan J, Gaddipati S, Suvas S, et al. miR-15a/16 reduces retinal leukostasis through decreased pro-inflammatory signaling. J Neuroinflammation. 2016;13(1):305.

30. Smith KM, Guerau-de-Arellano M, Costinean S, Williams JL, Bottoni A, Mavrikis Cox G, et al. miR-29ab1 deficiency identifies a negative feedback loop controlling Th1 bias that is dysregulated in multiple sclerosis. J Immunol Baltim Md 1950. 2012;189(4):1567-76.

31. Zhu E, Wang X, Zheng B, Wang Q, Hao J, Chen S, et al. miR-2ob suppresses Th17 differentiation and the pathogenesis of experimental autoimmune encephalomyelitis by targeting RORyt and STAT3. J Immunol. 2014;192(12):5599-609.

32. Zampetaki A, Kiechl S, Drozdov I, Willeit P, Mayr U, Prokopi M, et al. Plasma microRNA profiling reveals loss of endothelial miR-126 and other microRNAs in type 2 diabetes. Circ Res. 2010;107(6):810-7.
33. Raciti GA, Longo M, Parrillo L, Ciccarelli M, Mirra P, Ungaro P, et al. Understanding type 2 diabetes: from genetics to epigenetics. Acta Diabetol. 2015;52(5):821-7.

34. Godfrey KM, Sheppard A, Gluckman PD, Lillycrop KA, Burdge GC, McLean C, et al. Epigenetic gene promoter methylation at birth is associated with child's later adiposity. Diabetes. 2011;60(5):1528-34.

35. Toperoff G, Aran D, Kark JD, Rosenberg M, Dubnikov T, Nissan B, et al. Genome-wide survey reveals predisposing diabetes type 2-related DNA methylation variations in human peripheral blood. Hum Mo Genet. 2012;21(2):371-83.

36. Guo Y, Huang Z, Sang D, Gao Q, Li Q. The role of nutrition in the prevention and intervention of type 2 diabetes. Front Bioeng Biotechnol. 2020;8:575442.

37. Vo TX, Revesz A, Sohi G, Ma N, Hardy DB. Maternal protein restriction leads to enhanced hepatic gluconeogenic gene expression in adult male rat offspring due to impaired expression of the liver $\mathrm{X}$ receptor. J Endocrinol. 2013;218(1):85-97.

38. Zhang Q, Sun X, Xiao X, Zheng J, Li M, Yu M, et al. Dietary chromium restriction of pregnant mice changes the methylation status of hepatic genes involved with insulin signaling in adult male offspring. PLoS One. 2017;12(1):e0169889.

39. Takaya J, Iharada A, Okihana H, Kaneko K. Magnesium deficiency in pregnant rats alters methylation of specific cytosines in the hepatic hydroxysteroid dehydrogenase-2 promoter of the offspring. Epigenetics. 2011;6(5):573-8.

40. Takaya J, Iharada A, Okihana H, Kaneko K. A calcium-deficient diet in pregnant, nursing rats induces hypomethylation of specific cytosines in the 11ß-hydroxysteroid dehydrogenase-1 promoter in pup liver. Nutr Res N Y N. 2013;33(11):961-70.

41. Nitert MD, Dayeh T, Volkov P, Elgzyri T, Hall E, Nilsson E, et al. Impact of an exercise intervention on DNA methylation in skeletal muscle from first-degree relatives of patients with type 2 diabetes. Diabetes. 2012;61(12):3322-32.

42. Allen SC, Mamotte CDS. Pleiotropic and adverse effects of statins - Do epigenetics play a role? J Pharmacol Exp Ther. 2017;362(2):319-26.

43. Ochoa-Rosales C, Portilla-Fernandez E, Nano J, Wilson R, Lehne B, Mishra PP, et al. Epigenetic link between statin therapy and type 2 diabetes. Diabetes Care. 2020;43(4):875-84.

44. O'Connell TM, Markunas CA. DNA Methylation and microRNA-based biomarkers for risk of type 2 diabetes. Curr Diabetes Rev. 2015;12(1): 20-9.

45. Dobosz AM, Dziewulska A, Dobrzyń A. Spotlight on epigenetics as a missing link between obesity and type 2 diabetes. Postepy Biochem. 2018;64(2):157-65.

46. Efrat S. Beta-cell dedifferentiation in type 2 diabetes: Concise review. Stem Cells. 2019;37(10):1267-72.

47. Lu TT-H, Heyne S, Dror E, Casas E, Leonhardt L, Boenke T, et al. The polycomb-dependent epigenome controls $\beta$ cell dysfunction, dedifferentiation, and diabetes. Cell Metab. 2018;27(6):1294-1308.e7.

48. Handal T, Eiges R. Correction of heritable epigenetic defects using editing tools. Int J Mol Sci. 2021;22(8):3966.

49. Rothstein MA, Cai Y, Marchant GE. Ethical implications of epigenetics research. Nat Rev Genet. 2009;10(4):224. 